\title{
LIVING WITH COVID-19: A SCHOOL GOING CHILD'S PERSPECTIVE
}

\section{Medical Science \\ Avanee Dalmia \\ Vijay Kumar Tadia*}

\author{
Class XI Student, The Shri Ram School Moulsari, Gurugram
}

Senior Resident Administrator, All India Institute of Medical Sciences, New Delhi *Corresponding Author

Chairperson, COVID-19 IEC Committee, All India Institute of Medical Sciences, New Delhi

\section{ABSTRACT}

Introduction: The world is experiencing unprecedented challenges from COVID-19, the coronavirus pandemic. Children are less susceptible, yet their lives have changed in profound ways. They are hit the hardest by psychosocial impact of this pandemic. This is a universal crisis and the impact will be lifelong for some children. Being quarantined in homes and institutions can impose greater psychological burden than the physical sufferings that may be caused by the virus. Closure of schools, lack of outdoor activity, etc. can potentially create monotony, distress, impatience, annoyance and varied neuropsychiatric manifestations.

Aims and Objectives: The aim of this study was to understand the perspectives of school going children towards COVID-19 pandemic in order to create child centric communication to address their most important concerns.

Materials and Methods: A Cross-sectional and descriptive study was conducted during the month of May \& June 2020. The data was collected by Snowball sampling through a national level survey. The study population included all the school going children willing to participate in the study and this included children from private schools, government schools and schools for the specially abled.

Results: Respondents - About 7,000 responses were obtained from almost all states and union territories. A few international responses from countries such as Indonesia, USA, Australia, Japan, UK etc were also received. Most of the respondents were of age 14 or below with almost equal gender distribution. The top 5 responding regions were Delhi, Haryana, Uttar Pradesh, Odisha and Rajasthan.

Causes of spread - About half $(50 \%)$ of the respondents felt that either meeting an infected person or touching something that they had touched would lead to the spread of the virus. Most children felt that they can avoid catching the virus by:

1. Maintaining a 6 feet distance between one another

2. Washing hands regularly

3. Avoiding crowded areas.

Communication channels: The main channel of communication for information has been news channels for people in majority of the states, of all age groups as well as both genders. The second most used channel of communication has been social media for a few states and government advisories for others

Fears - Most of the respondents were scared of the fact that it is uncertain when COVID-19 will end while others were concerned over no cure being found as yet. This trend was similar between different age groups, genders and majority of the states.

Hopes - Children in most states and of both genders are mostly looking forward to being able to go to normal school once again while children above the age of 17 are looking forward to going out once the pandemic ends.

Conclusion: The children were aware of the basic facts about the virus, the mode of its spread, methods of prevention of the infection. They want to know when the pandemic would end, how to live with it, how an infected person could be cured and when the vaccine would be made available.

\section{KEYWORDS}

\section{INTRODUCTION:}

The world is experiencing unprecedented challenges from COVID-19, the coronavirus pandemic.(1)

Children are less susceptible, yet their lives have changed in profound ways. They are hit the hardest by psychosocial impact of this pandemic. This is a universal crisis and the impact will be lifelong for some children.(2)(3) Being quarantined in homes and institutions can impose greater psychological burden than the physical sufferings that may be caused by the virus. Closure of schools, lack of outdoor activity, etc. can potentially promote monotony, distress, impatience, annoyance and varied neuropsychiatric manifestations.(3)

The Children infected with COVID-19 have milder illness, low morbidity, better prognosis and probably lesser susceptibility (4)(5)(6)(7). Yet psychosocial impact is hue. The short-term and longlasting psychosocial impact is increasingly being recognized all over the world. (8) Being quarantined at home or elsewhere is a psychological burden and has varied neuropsychiatric manifestations and psychosocial stigma.(9) Confinement in home may cause immediate and lingering psychosocial impact on children due to drastic change in their lifestyle, physical activity and mental excursions.(8) The Children who get quarantined at institutions are the worst sufferers as support of their parents is not available.(10)

\section{AIM:}

The aim of this study was to understand the perspectives of school going children towards COVID-19 pandemic in order to create child centric communication to address their most important concerns.

\section{Objectives:}

1. Understand the impact of COVID-19 on school going children through an online survey.

2. Create child centric communication to address their most important concerns.

Methods:

Type of Study: Cross-sectional, descriptive

Study period: May - June 2020

Sampling Method: Snowball sampling through a national level survey

Study Population: All school going children willing to participate in the study. This included children from private schools, government schools and schools for the specially abled.

A survey questionnaire was formulated by brainstorming, which was followed by validation by experts. This questionnaire was then shared with respondents through Google Forms. Responses were obtained for a period of 2 months from May to June 2020. The responses were analyzed with the help of Microsoft Suite. 


\section{RESULTS:}

About 7,000 responses were obtained from almost all states and union territories in India - from Jammu \& Kashmir to Kerala and from Gujarat all the way to Arunachal Pradesh (Figure 1). A few international responses were also received from countries such as Indonesia, USA, Australia, Japan and UK (Table 3).

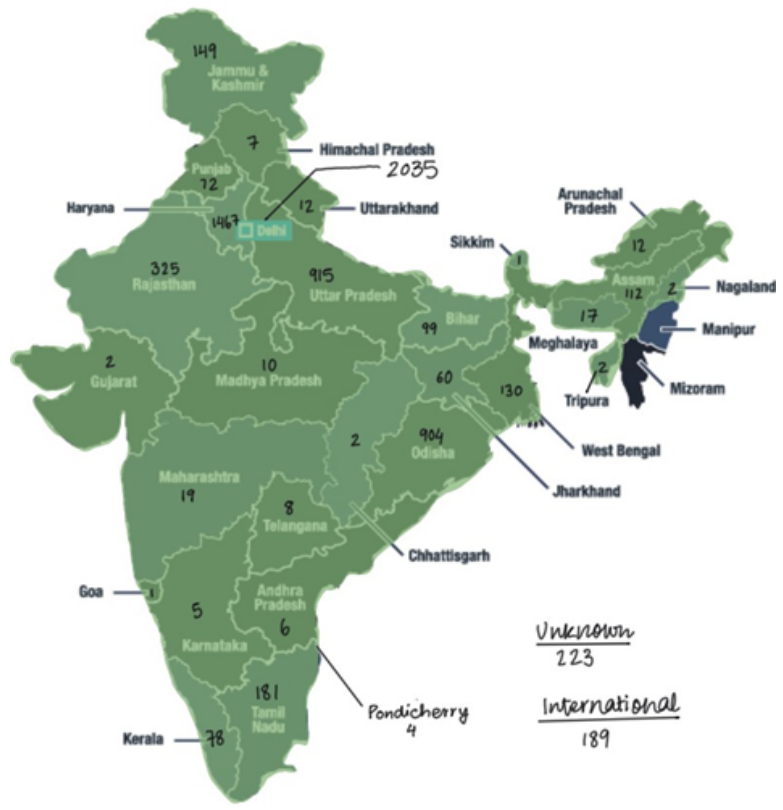

Figure 1. Geographical Distribution

The states highlighted in green were covered by the survey

Age \& Gender: Most respondents were of age 14 or below (Table 1A). The gender distribution of respondents was approximately 50-50 (Table 1B).

Table 1A: Age Distribution

\begin{tabular}{|c|c|}
\hline Age Group & Percentage \\
\hline Below 12 & $44 \%$ \\
\hline 12 to 14 & $31 \%$ \\
\hline 15 to 17 & $18 \%$ \\
\hline Above 17 & $7 \%$ \\
\hline Grand Total & $100 \%$ \\
\hline
\end{tabular}

\section{Table 1B: Gender Distribution}

\begin{tabular}{|l|l|l|l|}
\hline \multicolumn{4}{|c|}{ Age Groups and Gender } \\
\hline Age Group & Female & Male & Total \\
\hline Below 12 & $47 \%$ & $52 \%$ & $100 \%$ \\
\hline 12 to 14 & $53 \%$ & $46 \%$ & $100 \%$ \\
\hline 15 to 17 & $48 \%$ & $52 \%$ & $100 \%$ \\
\hline Above 17 & $38 \%$ & $62 \%$ & $100 \%$ \\
\hline Grand Total & $\mathbf{4 9 \%}$ & $\mathbf{5 1 \%}$ & $\mathbf{1 0 0 \%}$ \\
\hline
\end{tabular}

Respondents by Region (Tables 2A \& 2B)

The top 5 responding regions were (Table $2 \mathrm{~A}$ ):

1. Delhi

2. Haryana

3. Uttar Pradesh

4. Odisha

5. Rajasthan

The top 5 responding countries were (Table $2 \mathrm{~B}$ ):

1. India

2. Indonesia

3. Singapore

4. USA

5. United Kingdom

Table 2A: Respondents by State (within India)

\begin{tabular}{|l|l|l|l|}
\hline States/Union Territories & Female & Male & Total \\
\hline Delhi & 1091 & 927 & 2035 \\
\hline Haryana & 628 & 827 & 1467 \\
\hline
\end{tabular}

\begin{tabular}{|c|c|c|c|}
\hline Uttar Pradesh & 433 & 475 & 915 \\
\hline Odisha & 448 & 455 & 904 \\
\hline Rajasthan & 163 & 158 & 325 \\
\hline Other/Unkown & 108 & 111 & 223 \\
\hline Tamil Nadu & 84 & 95 & 181 \\
\hline Jammu \& Kashmir & 87 & 62 & 149 \\
\hline West Bengal & 48 & 82 & 130 \\
\hline Assam & 65 & 47 & 112 \\
\hline Bihar & 38 & 61 & 99 \\
\hline Kerala & 39 & 39 & 78 \\
\hline Punjab & 31 & 41 & 72 \\
\hline \begin{tabular}{|l} 
Jharkhand \\
\end{tabular} & 20 & 40 & 60 \\
\hline Maharashtra & 11 & 8 & 19 \\
\hline Meghalaya & 7 & 10 & 17 \\
\hline Uttarakhand & 5 & 5 & 12 \\
\hline Arunachal Pradesh & 3 & 9 & 12 \\
\hline Madhya Pradesh & 6 & 4 & 10 \\
\hline Telangana & 3 & 5 & 8 \\
\hline Himachal Pradesh & 1 & 6 & 7 \\
\hline Andhra Pradesh & 1 & 5 & 6 \\
\hline Karnataka & 4 & 1 & 5 \\
\hline Puducherry & 4 & 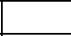 & 4 \\
\hline Tripura & & 2 & 2 \\
\hline Nagaland & 1 & 1 & 2 \\
\hline Gujarat & & 2 & 2 \\
\hline Chattisgarh & 2 & & 2 \\
\hline Goa & 1 & & 1 \\
\hline Sikkim & & 1 & 1 \\
\hline Total & 3332 & 3479 & 6860 \\
\hline \multicolumn{4}{|c|}{ Table 2B: Respondents by Country } \\
\hline Countries & Female & Male & Total \\
\hline India & 3332 & 3479 & 6860 \\
\hline Indonesia & 83 & 72 & 157 \\
\hline Singapore & & 10 & 11 \\
\hline USA & 2 & 5 & 10 \\
\hline United Kingdom & 3 & 1 & 5 \\
\hline Denmark & 1 & & 1 \\
\hline Australia & & 1 & 1 \\
\hline Korea & & 1 & 1 \\
\hline Nepal & & 1 & 1 \\
\hline Saudi Arabia & & 1 & 1 \\
\hline Dubai & & 1 & 1 \\
\hline Total & 3421 & 3572 & 7049 \\
\hline
\end{tabular}

What do you think are the main causes for the spread of the virus ? Around $50 \%$ of the respondents felt that either meeting an infected person or touching something that an infected person had touched would lead to the spread of the virus (Table 3)

Table 3: Causes for the spread of the virus

\begin{tabular}{|l|l|}
\hline Cause for the spread of Virus & Percentage \\
\hline $\begin{array}{l}\text { When you come in contact with a COVID-19 infected } \\
\text { person }\end{array}$ & 26 \\
\hline $\begin{array}{l}\text { When you touch something a COVID infected person } \\
\text { has touched }\end{array}$ & 21 \\
\hline Coughing and sneezing & 16 \\
\hline Being in a crowded area & 16 \\
\hline Low Immunity & 13 \\
\hline Eating Outside & 5 \\
\hline Drinking unfiltered water & 3 \\
\hline Total & $\mathbf{1 0 0}$ \\
\hline
\end{tabular}

According to you, what are the best measures to prevent yourself from catching the virus?

Most children feel that they can avoid catching the virus by maintaining 6 feet distance, washing hands regularly and avoiding crowded areas (Table 4).

Table 4: Measures to prevent yourself from catching the virus

\begin{tabular}{|l|l|}
\hline Measures to prevent yourself from catching the virus & Percentage \\
\hline
\end{tabular} \begin{tabular}{|l|l|}
\hline Washing your hands regularly & 21 \\
\hline
\end{tabular}

\begin{tabular}{|l|l|}
\hline Wearing a mask when you go out & 19 \\
\hline
\end{tabular}

\begin{tabular}{|l|l}
\hline Maintaining a distance of 6 feet & 14
\end{tabular}

Avoiding crowded areas 
Avoiding touching your face

Avoiding physical contact with outsiders

Self quarantine

Eating healthy

Checking your temperature regularly

Drinking hot beverages

Table 5A: Source of Knowledge (State wise)

\begin{tabular}{|c|c|c|c|c|c|c|c|c|}
\hline $5 \mathrm{~A}$ & & & & & urce & & & \\
\hline State & $\begin{array}{l}\text { Friends and } \\
\text { family }\end{array}$ & $\begin{array}{l}\text { Government } \\
\text { advisory }\end{array}$ & Internet & $\begin{array}{l}\text { News } \\
\text { Channels }\end{array}$ & School & Social media & Other & Total \\
\hline Delhi & $7 \%$ & $7 \%$ & $9 \%$ & $54 \%$ & $3 \%$ & $15 \%$ & $6 \%$ & $100 \%$ \\
\hline Haryana & $6 \%$ & $8 \%$ & $7 \%$ & $60 \%$ & $2 \%$ & $13 \%$ & $4 \%$ & $100 \%$ \\
\hline Uttar Pradesh & $14 \%$ & $8 \%$ & $11 \%$ & $45 \%$ & $4 \%$ & $13 \%$ & $5 \%$ & $100 \%$ \\
\hline Rajasthan & $3 \%$ & $14 \%$ & $6 \%$ & $53 \%$ & $1 \%$ & $16 \%$ & $8 \%$ & $100 \%$ \\
\hline Tamil Nadu & $2 \%$ & $8 \%$ & $2 \%$ & $65 \%$ & $4 \%$ & $15 \%$ & $6 \%$ & $100 \%$ \\
\hline Jammu \& Kashmir & $16 \%$ & $6 \%$ & $3 \%$ & $62 \%$ & $0 \%$ & $8 \%$ & $5 \%$ & $100 \%$ \\
\hline West Bengal & $6 \%$ & $12 \%$ & $18 \%$ & $39 \%$ & $0 \%$ & $21 \%$ & $5 \%$ & $100 \%$ \\
\hline Assam & $12 \%$ & $4 \%$ & $9 \%$ & $51 \%$ & $0 \%$ & $21 \%$ & $4 \%$ & $100 \%$ \\
\hline Bihar & $5 \%$ & $17 \%$ & $10 \%$ & $49 \%$ & $0 \%$ & $16 \%$ & $2 \%$ & $100 \%$ \\
\hline Kerala & $9 \%$ & $15 \%$ & $5 \%$ & $45 \%$ & $0 \%$ & $24 \%$ & $1 \%$ & $100 \%$ \\
\hline Jharkhand & $2 \%$ & $5 \%$ & $10 \%$ & $62 \%$ & $0 \%$ & $18 \%$ & $3 \%$ & $100 \%$ \\
\hline Maharashtra & $21 \%$ & $16 \%$ & $5 \%$ & $32 \%$ & $11 \%$ & $11 \%$ & $5 \%$ & $100 \%$ \\
\hline Meghalaya & $24 \%$ & $6 \%$ & $6 \%$ & $47 \%$ & $0 \%$ & $18 \%$ & $0 \%$ & $100 \%$ \\
\hline Uttarakhand & $0 \%$ & $25 \%$ & $0 \%$ & $33 \%$ & $8 \%$ & $25 \%$ & $8 \%$ & $100 \%$ \\
\hline Arunachal Pradesh & $17 \%$ & $8 \%$ & $0 \%$ & $50 \%$ & $0 \%$ & $8 \%$ & $17 \%$ & $100 \%$ \\
\hline Madhya Pradesh & $10 \%$ & $20 \%$ & $20 \%$ & $20 \%$ & $0 \%$ & $20 \%$ & $10 \%$ & $100 \%$ \\
\hline Telangana & $0 \%$ & $0 \%$ & $25 \%$ & $75 \%$ & $0 \%$ & $0 \%$ & $0 \%$ & $100 \%$ \\
\hline Himachal Pradesh & $0 \%$ & $0 \%$ & $14 \%$ & $86 \%$ & $0 \%$ & $0 \%$ & $0 \%$ & $100 \%$ \\
\hline Andhra Pradesh & $0 \%$ & $33 \%$ & $0 \%$ & $33 \%$ & $0 \%$ & $17 \%$ & $17 \%$ & $100 \%$ \\
\hline Karnataka & $20 \%$ & $20 \%$ & $40 \%$ & $20 \%$ & $0 \%$ & $0 \%$ & $0 \%$ & $100 \%$ \\
\hline Puducherry & $0 \%$ & $25 \%$ & $0 \%$ & $25 \%$ & $0 \%$ & $50 \%$ & $0 \%$ & $100 \%$ \\
\hline Tripura & $100 \%$ & $0 \%$ & $0 \%$ & $0 \%$ & $0 \%$ & $0 \%$ & $0 \%$ & $100 \%$ \\
\hline Gujarat & $50 \%$ & $0 \%$ & $0 \%$ & $50 \%$ & $0 \%$ & $0 \%$ & $0 \%$ & $100 \%$ \\
\hline Goa & $0 \%$ & $0 \%$ & $0 \%$ & $0 \%$ & $100 \%$ & $0 \%$ & $0 \%$ & $100 \%$ \\
\hline Sikkim & $0 \%$ & $100 \%$ & $0 \%$ & $0 \%$ & $0 \%$ & $0 \%$ & $0 \%$ & $100 \%$ \\
\hline Other/Unkown & $8 \%$ & $7 \%$ & $11 \%$ & $45 \%$ & $5 \%$ & $16 \%$ & $8 \%$ & $100 \%$ \\
\hline Total & $8 \%$ & $9 \%$ & $9 \%$ & $53 \%$ & $2 \%$ & $15 \%$ & $5 \%$ & $100 \%$ \\
\hline
\end{tabular}

Table 5B. Source of Knowledge (Age wise)

\begin{tabular}{|c|c|c|c|c|c|c|c|c|c|}
\hline $5 B$ & & & & & Source & & & & \\
\hline Age Group & Doctors & $\begin{array}{l}\text { Friends and } \\
\text { family }\end{array}$ & $\begin{array}{l}\text { Government } \\
\text { advisory }\end{array}$ & Internet & $\begin{array}{l}\text { News } \\
\text { Channels }\end{array}$ & School & $\begin{array}{l}\text { Social } \\
\text { media }\end{array}$ & Other & Total \\
\hline Below 12 & $3 \%$ & $11 \%$ & $9 \%$ & $6 \%$ & $56 \%$ & $2 \%$ & $12 \%$ & $1 \%$ & $100 \%$ \\
\hline 12 to 14 & $4 \%$ & $7 \%$ & $8 \%$ & $9 \%$ & $53 \%$ & $3 \%$ & $15 \%$ & $1 \%$ & $100 \%$ \\
\hline 15 to 17 & $3 \%$ & $5 \%$ & $7 \%$ & $14 \%$ & $48 \%$ & $2 \%$ & $20 \%$ & $1 \%$ & $100 \%$ \\
\hline Above 17 & $5 \%$ & $1 \%$ & $18 \%$ & $12 \%$ & $44 \%$ & $1 \%$ & $17 \%$ & $1 \%$ & $100 \%$ \\
\hline Total & $4 \%$ & $8 \%$ & $9 \%$ & $9 \%$ & $53 \%$ & $2 \%$ & $15 \%$ & $1 \%$ & $100 \%$ \\
\hline
\end{tabular}

Table 5C. Source of Knowledge (Gender wise)

From where have you learnt the most about COVID-19 from?

\begin{tabular}{|l|l|l|l|l|}
\hline $\mathbf{5 C}$ & \multicolumn{5}{|l|}{} \\
\hline Gender & Doctors & $\begin{array}{l}\text { Friends and } \\
\text { family }\end{array}$ & $\begin{array}{l}\text { Government } \\
\text { advisory }\end{array}$ & Internet \\
\hline Female & $4 \%$ & $8 \%$ & $9 \%$ & $8 \%$ \\
\hline Male & $4 \%$ & $7 \%$ & $9 \%$ & $9 \%$ \\
\hline Total & $4 \%$ & $8 \%$ & $9 \%$ & $9 \%$ \\
\hline
\end{tabular}

What about COVID-19 makes you nervous or scared?

Most respondents were scared of the fact that it is uncertain when

COVID-19 will end while others were concerned over no cure being found as yet. This trend was similar between majority of the states (Table 6A), different age groups (Table 6B) and genders (Table 6C).

Table 6A: Reason for Fear (State wise)

\begin{tabular}{|l|l|l|l|l|l|l|}
\hline 6A & \multicolumn{5}{|c|}{ Reason } \\
\hline State & $\begin{array}{l}\text { I don't } \\
\text { know } \\
\text { enough } \\
\text { about it }\end{array}$ & $\begin{array}{l}\text { I don't } \\
\text { know } \\
\text { when it } \\
\text { will end }\end{array}$ & $\begin{array}{l}\text { I might } \\
\text { get it }\end{array}$ & $\begin{array}{l}\text { There' } \\
\text { s no } \\
\text { cure }\end{array}$ & Other & Total \\
\hline Delhi & $9 \%$ & $42 \%$ & $12 \%$ & $33 \%$ & $4 \%$ & $100 \%$ \\
\hline
\end{tabular}

All the above

Total 100

Where have you learnt the most about COVID-19 from?

The main channel of communication for information has been news channels for people in majority of the states (Table 5A), of all age groups (Table 5B) as well as both genders (Table 5C). The second most used channel of communication has been social media for a few states and government advisories for others. 


\begin{tabular}{|l|l|l|l|l|l|l|}
\hline Meghalaya & $0 \%$ & $59 \%$ & $6 \%$ & $29 \%$ & $6 \%$ & $100 \%$ \\
\hline Uttarakhand & $8 \%$ & $67 \%$ & $0 \%$ & $25 \%$ & $0 \%$ & $100 \%$ \\
\hline Arunachal Pradesh & $0 \%$ & $58 \%$ & $17 \%$ & $25 \%$ & $0 \%$ & $100 \%$ \\
\hline Madhya Pradesh & $0 \%$ & $70 \%$ & $0 \%$ & $30 \%$ & $0 \%$ & $100 \%$ \\
\hline Telangana & $25 \%$ & $13 \%$ & $0 \%$ & $63 \%$ & $0 \%$ & $100 \%$ \\
\hline Himachal Pradesh & $0 \%$ & $71 \%$ & $0 \%$ & $29 \%$ & $0 \%$ & $100 \%$ \\
\hline Andhra Pradesh & $0 \%$ & $50 \%$ & $17 \%$ & $17 \%$ & $17 \%$ & $100 \%$ \\
\hline Karnataka & $0 \%$ & $60 \%$ & $0 \%$ & $40 \%$ & $0 \%$ & $100 \%$ \\
\hline Puducherry & $0 \%$ & $75 \%$ & $0 \%$ & $25 \%$ & $0 \%$ & $100 \%$ \\
\hline Tripura & $50 \%$ & $0 \%$ & $0 \%$ & $50 \%$ & $0 \%$ & $100 \%$ \\
\hline Nagaland & $0 \%$ & $50 \%$ & $50 \%$ & $0 \%$ & $0 \%$ & $100 \%$ \\
\hline Gujarat & $0 \%$ & $0 \%$ & $0 \%$ & $50 \%$ & $50 \%$ & $100 \%$ \\
\hline Chattisgarh & $0 \%$ & $0 \%$ & $0 \%$ & $100 \%$ & $0 \%$ & $100 \%$ \\
\hline Goa & $0 \%$ & $100 \%$ & $0 \%$ & $0 \%$ & $0 \%$ & $100 \%$ \\
\hline Other/Unkown & $6 \%$ & $39 \%$ & $10 \%$ & $38 \%$ & $7 \%$ & $100 \%$ \\
\hline Total & $6 \%$ & $41 \%$ & $12 \%$ & $37 \%$ & $4 \%$ & $100 \%$ \\
\hline
\end{tabular}

Table 6B: Reason for Fear (Age wise)

\begin{tabular}{|l|l|l|l|l|l|l|}
\hline 6B & Reason \\
\hline $\begin{array}{l}\text { Age } \\
\text { Group }\end{array}$ & $\begin{array}{l}\text { I don't know } \\
\text { enough } \\
\text { about it }\end{array}$ & $\begin{array}{l}\text { I don't } \\
\text { know when } \\
\text { it will end }\end{array}$ & $\begin{array}{l}\text { I might } \\
\text { get it }\end{array}$ & $\begin{array}{l}\text { There's } \\
\text { no cure }\end{array}$ & Other & Total \\
\hline Below 12 & $6 \%$ & $40 \%$ & $14 \%$ & $38 \%$ & $3 \%$ & $100 \%$ \\
\hline 12 to 14 & $6 \%$ & $40 \%$ & $13 \%$ & $37 \%$ & $4 \%$ & $100 \%$ \\
\hline 15 to 17 & $8 \%$ & $44 \%$ & $9 \%$ & $34 \%$ & $6 \%$ & $100 \%$ \\
\hline Above 17 & $5 \%$ & $41 \%$ & $13 \%$ & $37 \%$ & $3 \%$ & $100 \%$ \\
\hline Total & $6 \%$ & $41 \%$ & $12 \%$ & $37 \%$ & $4 \%$ & $100 \%$ \\
\hline
\end{tabular}

Table 6C: Reason for Fear (Gender wise)

\begin{tabular}{|l|l|l|l|l|l|l|}
\hline 6C & \multicolumn{5}{|c|}{ Reason } \\
\hline Gender & $\begin{array}{l}\text { I don't know } \\
\text { enough } \\
\text { about it }\end{array}$ & $\begin{array}{l}\text { I don't know } \\
\text { when it will } \\
\text { end }\end{array}$ & $\begin{array}{l}\text { I } \\
\text { might } \\
\text { get it }\end{array}$ & $\begin{array}{l}\text { There's } \\
\text { no cure }\end{array}$ & Other & Total \\
\hline Female & $6 \%$ & $41 \%$ & $13 \%$ & $37 \%$ & $4 \%$ & $100 \%$ \\
\hline Male & $7 \%$ & $41 \%$ & $12 \%$ & $36 \%$ & $4 \%$ & $100 \%$ \\
\hline Total & $6 \%$ & $41 \%$ & $12 \%$ & $37 \%$ & $4 \%$ & $100 \%$ \\
\hline
\end{tabular}

\section{What do you look forward to most after this pandemic ends?}

Children in most states (Table $7 \mathrm{~A}$ )and of both genders (Table $7 \mathrm{C}$ ) are mostly looking forward to being able to go to normal school once again while people above the age of 17 (Table 7B) are looking forward to going out once the pandemic ends.

Table 7A. What respondents are looking forward to (State wise)

\begin{tabular}{|l|l|l|l|l|l|l|}
\hline 7A & \multicolumn{5}{|c|}{ Activity } \\
\hline State & $\begin{array}{l}\text { Being } \\
\text { able to } \\
\text { go out }\end{array}$ & $\begin{array}{l}\text { Eating } \\
\text { outside } \\
\text { food }\end{array}$ & $\begin{array}{l}\text { Meeting } \\
\text { friends }\end{array}$ & $\begin{array}{l}\text { Normal } \\
\text { school }\end{array}$ & Other & Total \\
\hline Delhi & $26 \%$ & $6 \%$ & $23 \%$ & $39 \%$ & $6 \%$ & $100 \%$ \\
\hline Haryana & $27 \%$ & $3 \%$ & $22 \%$ & $40 \%$ & $8 \%$ & $100 \%$ \\
\hline Uttar Pradesh & $29 \%$ & $5 \%$ & $23 \%$ & $38 \%$ & $5 \%$ & $100 \%$ \\
\hline Odisha & $26 \%$ & $2 \%$ & $12 \%$ & $56 \%$ & $4 \%$ & $100 \%$ \\
\hline Rajasthan & $29 \%$ & $7 \%$ & $14 \%$ & $44 \%$ & $6 \%$ & $100 \%$ \\
\hline Tamil Nadu & $31 \%$ & $4 \%$ & $19 \%$ & $41 \%$ & $5 \%$ & $100 \%$ \\
\hline $\begin{array}{l}\text { Jammu \& } \\
\text { Kashmir }\end{array}$ & $26 \%$ & $5 \%$ & $17 \%$ & $50 \%$ & $1 \%$ & $100 \%$ \\
\hline West Bengal & $32 \%$ & $6 \%$ & $25 \%$ & $28 \%$ & $8 \%$ & $100 \%$ \\
\hline Assam & $27 \%$ & $3 \%$ & $16 \%$ & $51 \%$ & $4 \%$ & $100 \%$ \\
\hline Bihar & $28 \%$ & $7 \%$ & $17 \%$ & $42 \%$ & $5 \%$ & $100 \%$ \\
\hline Kerala & $35 \%$ & $4 \%$ & $21 \%$ & $38 \%$ & $3 \%$ & $100 \%$ \\
\hline Punjab & $26 \%$ & $4 \%$ & $29 \%$ & $35 \%$ & $6 \%$ & $100 \%$ \\
\hline Jharkhand & $32 \%$ & $8 \%$ & $22 \%$ & $37 \%$ & $2 \%$ & $100 \%$ \\
\hline Maharashtra & $26 \%$ & $5 \%$ & $37 \%$ & $32 \%$ & $0 \%$ & $100 \%$ \\
\hline Meghalaya & $29 \%$ & $0 \%$ & $12 \%$ & $53 \%$ & $6 \%$ & $100 \%$ \\
\hline Uttarakhand & $17 \%$ & $0 \%$ & $8 \%$ & $75 \%$ & $0 \%$ & $100 \%$ \\
\hline $\begin{array}{l}\text { Arunachal } \\
\text { Pradesh }\end{array}$ & $42 \%$ & $0 \%$ & $8 \%$ & $50 \%$ & $0 \%$ & $100 \%$ \\
\hline Madhya Pradesh & $0 \%$ & $0 \%$ & $20 \%$ & $70 \%$ & $10 \%$ & $100 \%$ \\
\hline Telangana & $38 \%$ & $13 \%$ & $25 \%$ & $0 \%$ & $25 \%$ & $100 \%$ \\
\hline Himachal Pradesh & $29 \%$ & $0 \%$ & $14 \%$ & $57 \%$ & $0 \%$ & $100 \%$ \\
\hline Andhra Pradesh & $0 \%$ & $17 \%$ & $17 \%$ & $50 \%$ & $17 \%$ & $100 \%$ \\
\hline Karnataka & $40 \%$ & $0 \%$ & $0 \%$ & $40 \%$ & $20 \%$ & $100 \%$ \\
\hline Puducherry & $50 \%$ & $0 \%$ & $25 \%$ & $25 \%$ & $0 \%$ & $100 \%$ \\
\hline Tripura & $0 \%$ & $0 \%$ & $50 \%$ & $50 \%$ & $0 \%$ & $100 \%$ \\
\hline Nagaland & $0 \%$ & $0 \%$ & $0 \%$ & $100 \%$ & $0 \%$ & $100 \%$ \\
\hline Gujarat & $0 \%$ & $50 \%$ & $50 \%$ & $0 \%$ & $0 \%$ & $100 \%$ \\
\hline & & & & & & \\
\hline
\end{tabular}

\begin{tabular}{|l|l|l|l|l|l|l|}
\hline Chattisgarh & $100 \%$ & $0 \%$ & $0 \%$ & $0 \%$ & $0 \%$ & $100 \%$ \\
\hline Goa & $0 \%$ & $0 \%$ & $0 \%$ & $100 \%$ & $0 \%$ & $100 \%$ \\
\hline Sikkim & $0 \%$ & $0 \%$ & $0 \%$ & $100 \%$ & $0 \%$ & $100 \%$ \\
\hline Other/Unkown & $23 \%$ & $9 \%$ & $21 \%$ & $40 \%$ & $7 \%$ & $100 \%$ \\
\hline Total & $27 \%$ & $5 \%$ & $21 \%$ & $41 \%$ & $6 \%$ & $100 \%$ \\
\hline
\end{tabular}

Table 7B. What respondents are looking forward to (Age wise) What do you look forward to most after this pandemic ends?

\begin{tabular}{|l|l|l|l|l|l|l|}
\hline 7B & \multicolumn{5}{|c|}{ Activity } \\
\hline Age Group & $\begin{array}{l}\text { Being } \\
\text { able to } \\
\text { go out }\end{array}$ & $\begin{array}{l}\text { Eating } \\
\text { outside } \\
\text { food }\end{array}$ & $\begin{array}{l}\text { Meeting } \\
\text { friends }\end{array}$ & $\begin{array}{l}\text { Normal } \\
\text { school }\end{array}$ & Other & Total \\
\hline Below 12 & $28 \%$ & $3 \%$ & $18 \%$ & $45 \%$ & $6 \%$ & $100 \%$ \\
\hline 12 to 14 & $25 \%$ & $6 \%$ & $23 \%$ & $40 \%$ & $6 \%$ & $100 \%$ \\
\hline 15 to 17 & $28 \%$ & $4 \%$ & $25 \%$ & $37 \%$ & $5 \%$ & $100 \%$ \\
\hline Above 17 & $36 \%$ & $4 \%$ & $19 \%$ & $32 \%$ & $9 \%$ & $100 \%$ \\
\hline Total & $27 \%$ & $5 \%$ & $21 \%$ & $41 \%$ & $6 \%$ & $100 \%$ \\
\hline
\end{tabular}

Table 7c. What respondents are looking forward to (Gender wise)

\begin{tabular}{|l|l|l|l|l|l|l|}
\hline 7C & \multicolumn{7}{|c|}{ Activity } \\
\hline Gender & $\begin{array}{l}\text { Being } \\
\text { able to } \\
\text { go out }\end{array}$ & $\begin{array}{l}\text { Eating } \\
\text { outside } \\
\text { food }\end{array}$ & $\begin{array}{l}\text { Meeting } \\
\text { friends }\end{array}$ & $\begin{array}{l}\text { Normal } \\
\text { school }\end{array}$ & Other & Total \\
\hline Female & $28 \%$ & $4 \%$ & $20 \%$ & $42 \%$ & $6 \%$ & $100 \%$ \\
\hline Male & $27 \%$ & $5 \%$ & $22 \%$ & $40 \%$ & $6 \%$ & $100 \%$ \\
\hline Total & $27 \%$ & $5 \%$ & $21 \%$ & $41 \%$ & $6 \%$ & $100 \%$ \\
\hline
\end{tabular}

\section{CONCLUSIONS \& DISCUSSION}

What the children were aware of

- Basic facts about the virus

- How it is spread

- How to prevent the infection

- Social distancing and the message of 'stay home stay safe

\section{Want Children wanted to know}

- When it would en

- How to live with it

- How an infected person could be cured

- When the vaccine would be made

- How they could keep themselves and their families safe

What children were affected by:

- Not being able to meet friend

- Having to learn using online platforms

\section{REFERENCES:}

1. Decisive Action in an Unprecedented Crisis [Internet]. The World Bank. 2020 [cited 2020 Aug 2]. Available from: https:// www. world bank. org/en/ news/ feature 2020/04/17/decisive-action-in-an-unprecedented-crisis

2. UNICEF. COVID-19 and children [Internet]. [cited 2020 Aug 2]. Available from: https://data uniceforg/topic/covid-19-and-children/

3. Ghosh R, Dubey MJ, Chatterjee S, Dubey S. Impact of COVID -19 on children: special focus on the psychosocial aspect. Minerva Pediatr. 2020;72(3):226-35.

4. Tezer H, Bedir Demirdağ T. Novel coronavirus disease (Covid-19) in children [Internet]. Vol. 50, Turkish Journal of Medical Sciences. Turkiye Klinikleri; 2020 [cited 2020 Aug 2].p. 592-603. Available from: https://pubmed.ncbi.nlm.nih.gov/32304191/ Su L, Ma X, Yu H, Zhang Z, Bian P, Han Y, et al. The different clinical characteristics of corona virus disease cases between children and their families in China-the character of children with COVID-19. Emerg Microbes Infect [Internet]. 2020 Jan 1 [cited 2020 Aug 2]:9(1):707-13. Available from: $h$ ttps://pubmed ncbi nlm nih gov/32208917/

6. Ludvigsson JF. Systematic review of COVID-19 in children shows milder cases and better prognosis than adults [Internet]. Vol. 109, Acta Paediatrica, International Journal better prognosis than adults [Internet]. Vol. 109, Acta Paediatrica, International Journal of Paediatrics. Blackwell Publishing Ltd; 2020 [cited 2020 Aug 2]. p.
Available from: https://onlinelibrary.wiley.com/doi/full/10.1111/apa.15270

Dong Y, Dong Y, Mo X, Hu Y, Qi X, Jiang F, et al. Epidemiology of COVID-19 among children in China [Internet]. Vol. 145, Pediatrics. American Academy of Pediatrics; 2020 [cited 2020 Aug 2]. p. 20200702. Available from: https://doi.org/10.1542/peds.20200702

8. Wang G, Zhang Y, Zhao J, Zhang J, Jiang F. Mitigate the effects of home confinement on children during the COVID-19 outbreak [Internet]. Vol. 395, The Lancet. Lancet Publishing Group; 2020 [cited 2020 Aug 2]. p. 945-7. Available from: Publishing Group; 2020 [cited 2020/Aug 2]. p.

9. Brooks SK, Webster RK, Smith LE, Woodland L, Wessely S, Greenberg N, et al. Rapid Review The psychological impact of quarantine and how to reduce it: rapid review of the evidence. Lancet [Internet]. 2020 [cited 2020 Aug 2];395:912-20. Available from: https://doi.org/10.1016

10. Liu JJ, Bao Y, Huang X, Shi J, Lu L. Mental health considerations for children quarantined because of COVID-19 [Internet]. Vol. 4, The Lancet Child and Adolescent Health. Elsevier B.V.; 2020 [cited 2020 Aug 2]. p. 347-9. Available from: https://www.ncbi.nlm.nih.gov/pmc/articles/PMC7118598 